\title{
Tear analysis as the next routine body fluid test
}

\author{
Anis Barmada ${ }^{1,2} \cdot$ Scott A. Shippy $\mathbb{B}^{1,3}$
}

Received: 28 March 2020 / Revised: 16 April 2020 / Accepted: 22 April 2020 / Published online: 6 May 2020

(c) The Royal College of Ophthalmologists 2020

Among biological fluids like blood and urine, tear fluid is perhaps one of the most understated in terms of clinical value. Toward the age of personalized, preventive medicine and healthcare technology, the analysis of tears has a unique set of advantages to become the next comprehensive, routine body fluid test-in the clinic and beyond. To this end, recent breakthroughs as well as critical analytical and biochemical challenges are discussed.

Tears are the transparent, three-layered fluid covering the surface of the eye. For this avascular corneal surface, tears are responsible for those same roles that blood plays throughout the body-delivering oxygen and nutrients, removing waste, protecting against pathogens, and healing damage to the ocular surface. Importantly, tears also contain various electrolytes, proteins, lipids, and small molecule metabolites that are in constant exchange with ocular surface epithelial cells, reflecting corneal biochemistry and physiology. It is therefore natural for tear analysis to be of substantial significance for monitoring ocular health and disease [1]. However, the importance and value of tear analysis extend much beyond the ocular surface and eye.

From one aspect, tears are mainly secreted by the lacrimal glands in the eyelids through filtration from blood plasmawhich, itself, circulates around the entire body contacting all organs and tissues. Thus, tears are rich in clinical information of relevance to seemingly unrelated body parts. This potential of tears has been documented in the literature as a source for external drug screening and pharmacokinetic studies [2], as well as for biomarkers of various systemic

Scott A. Shippy

sshippy@uic.edu

1 Department of Chemistry, University of Illinois at Chicago, Chicago, IL, USA

2 Department of Ophthalmology and Visual Sciences, University of Illinois at Chicago, Chicago, IL, USA

3 Laboratory of Integrative Neuroscience, University of Illinois at Chicago, Chicago, IL, USA diseases—with and without ocular complications-including cancer, multiple sclerosis, diabetes mellitus, Parkinson's disease, cystic fibrosis, and Alzheimer's disease [3, 4]. More recently, a diagnostic company, Ascendant Dx, has been developing a tear protein-based breast cancer screening test, which was said to have promising results surpassing currently used imaging techniques [5].

From another aspect, the ocular surface is directly exposed to the outside environment, thereby making tear composition incredibly sensitive to external environmental changes. One recent study reported significant differences in tear lipid composition in two human populations with different exposure to particulate matter in urban and industrial zones [6], which can be valuable in characterizing environmental pollution and its impact on disease. In addition, tear chemical composition is sensitive to internal emotional and psychological states. The authors have previously developed a tear analysis method showing that emotional tears have a distinct chemical concentration compared to basal tears [7]. Most recently in the published literature, a prototyped design of a handheld sensor with disposable test strips for rapid tear cortisol quantitation was proposed to monitor physiological, occupational, and emotional stress [8].

Taken together, these observations suggest that tear analysis can provide important insights into internal pathophysiological changes and external drug intake, and beyond into environmental, circadian, psychological, and social states. If both tears and blood can provide similar clinical information, then a logical question needs to be answered: why should tears—not just blood—be analyzed?

The most notable advantage of the analysis of tears over blood is the minimal invasiveness in collection. Nanoliters to microliters of tears can be obtained practically noninvasively from the surface of the eye within seconds for rapid and continuous monitoring of health inside the clinic and outside through point-of-care devices. One up-and-coming tearbased biomarker technology employs smart contact lenses to sense disease-related tear biochemical changes in real time, which can be coupled with an embedded drug reservoir [9]. The technology is currently undergoing advancement for 


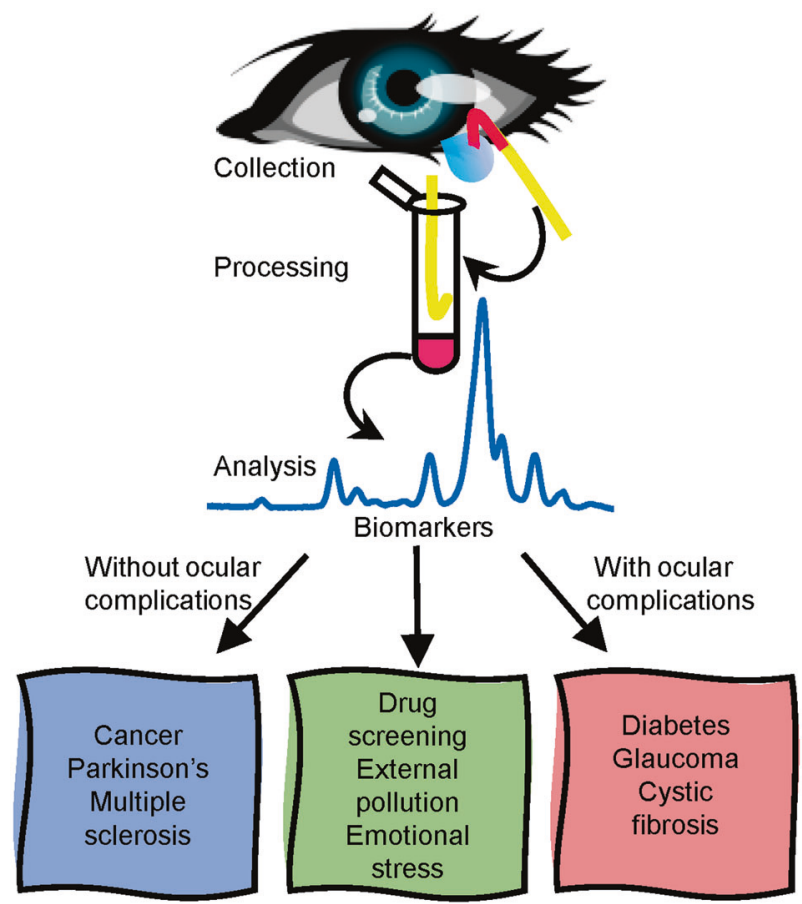

Fig. 1 Schematic diagram exemplifying a basic method for tears collection and analysis with clinical insights. Nanoliters of tears are collected via capillary action on phenol red thread with a color change length corresponding to the volume collected. Tears are then eluted and extracted from the thread to be analyzed using various technologies, including capillary electrophoresis and mass spectrometry. Different collection and analysis techniques based on similar principles, such as Schirmer strip and glass microcapillary tube, are also widely used.

biomarkers of a variety of medical conditions across research laboratories in both the public and private sectors, which can change the way health is monitored and healthcare is delivered by unlocking a whole new dimension of preventive, personalized medicine and healthcare technology [10]. To this end, further exploiting the potential in tear analysis should be a prominent goal on the ophthalmic research radar. Interestingly, a pilot study on the investigation of tear biomarker as an indicator of systemic health in astronauts, especially in relation to spaceflight environment and microgravity, has been proposed by the National Aeronautics and Space Administration [5].

The schematic diagram in Fig. 1 demonstrates a commonly used process for tears collection and analysis with potential clinical conclusions. However, critical analytical and biochemical challenges must be overcome in order to implement tear analysis in routine healthcare delivery. Methods of tear collection, storage, extraction, and preparation, as well as their unique impacts on analysis, must be standardized across research and clinical studies-like the long-term standardization efforts with blood. Simultaneously, the optimization of analytical technologies is needed in order to extract greater amounts of information from tear samples, such as advancing smart contact lenses to sense many tear biomarkers at once. One particular analytical challenge concerns the much lower concentration of some molecules in tears compared with blood, such as glucose and cortisol. Recently, the authors have developed a technique allowing the first-ever metabolite analysis of mouse tears [11], overcoming an important analytical challenge that has been long facing tear studies of models of human disease. These analytical challenges are coupled with biochemical challenges relating to drawing meaningful clinical conclusions from the generated tear molecular profiles. Similar to blood, tear biomarkers must be identified, and their levels correlated with the specific stage of disease or condition. Currently, tear metabolite characterization is barely present in the Human Metabolome Database as part of the Human Metabolome Project [12].

Making routine tear fluid testing a reality will take combined efforts from basic analytical scientists and ophthalmologists in order to accelerate the development and advancement of analytical technologies as well as their integration into the clinic. Naturally, such efforts are strengthened by extensive collaborations between analytical scientists and physicians, as well as by physician-scientists who are trained to recognize clinical limitations as compelling opportunities for scientific discovery [13]. Given the tremendous clinical advantage in tears and the pace of ophthalmic discovery, we hypothesize that the analysis of tears will be a routine test to monitor health in clinics and beyond within a decade or two.

\section{Compliance with ethical standards}

Conflict of interest The authors declare that they have no conflict of interest.

Publisher's note Springer Nature remains neutral with regard to jurisdictional claims in published maps and institutional affiliations.

\section{References}

1. Zhou L, Beuerman RW. Tear analysis in ocular surface diseases. Prog Retin Eye Res. 2012;31:527-50.

2. Raju KSR, Taneja I, Singh SP, Wahajuddin. Utility of noninvasive biomatrices in pharmacokinetic studies. Biomed Chromatogr. 2013;27:1354-66.

3. Hagan S, Martin E, Enríquez-de-Salamanca A. Tear fluid biomarkers in ocular and systemic disease: potential use for predictive, preventive and personalised medicine. EPMA J. 2016;7:15.

4. Kalló G, Emri M, Varga Z, Ujhelyi B, Tozsér J, Csutak A, et al. Changes in the chemical barrier composition of tears in Alzheimer's disease reveal potential tear diagnostic biomarkers. PLoS ONE. 2016;11:e0158000.

5. Morton S, Crucian B, Hagan S, Satyamitra M, Daily, A. Pilot study on the investigation of tear fluid biomarkers as an indicator of ocular, neurological, and immunological health in astronauts. 
NASA Technical Reports Server. 2018. U.S. National Aeronautics and Space Administration, Washington, DC. (abstract JSC-CN40616 \& JSC-CN-40620).

6. Gutierrez MdA, Colman Lerner JE, Giuliani DS, Porta AA, Andrinolo D. Comparative study of tear lipid composition in two human populations with different exposure to particulate matter in La Plata, Argentina. Environ Sci Pollut Res. 2019;26:6948-56.

7. Avilov V, Zeng Q, Shippy SA. Threads for tear film collection and support in quantitative amino acid analysis. Anal Bioanal Chem. 2016;408:5309-17.

8. Cardinell BA, Spano ML, La Belle JT. Toward a label-free electrochemical impedance immunosensor design for quantifying cortisol in tears. Crit Rev Biomed Eng. 2019;47:207-15.
9. Kim J, Cha E, Park JU. Recent advances in smart contact lenses. Adv Mater Technol. 2020;5:1900728.

10. Kim J, Campbell AS, de Ávila BE, Wang J. Wearable biosensors for healthcare monitoring. Nat Biotechnol. 2019;37:389-406.

11. Barmada A, Shippy SA. Thread-based assay for quantitative small molecule analysis of mice tear fluid by capillary electrophoresis. Anal Bioanal Chem. 2019;411:329-38.

12. Wishart DS, Feunang YD, Marcu A, Guo AC, Liang K, VázquezFresno R, et al. HMDB 4.0: the human metabolome database for 2018. Nucleic Acids Res. 2018;46:D608-D617.

13. Jain MK, Cheung VG, Utz PJ, Kobilka BK, Yamada T, Lefkowitz R. Saving the endangered physician-scientist - a plan for accelerating medical breakthroughs. N Engl J Med. 2019;381:399-402. 